\title{
Electrical Conductivity: Classical Electron and Quantum Mechanical Approaches
}

\author{
*Krishna Raj Adhikari \\ Pashchimanchal Campus, IOE/TU, Nepal \\ Department de Fisica Aplicada, Universitat Politecnica de Valencia, Spain \\ Email: adhikarikrishna@wrc.edu.np
}

\begin{abstract}
This article presents some review and annotations about classical electron and the quantum mechanical approaches to describe the electrical conductivity of materials specially metals.
\end{abstract}

Keywords: Electrical conductivity; drift velocity; Drude model; velocity space; relaxation time

\section{INTRODUCTION}

The concept of static electricity came first into the field of electricity after the work of Thales of Miletus' around $600 \mathrm{BC}$ when amber having been rubbed with a piece of cloth that had attracted the light particles and feathers. Thus it is believed that the word 'electricity' in English has its root in the Greek word 'elektron' which means amber ${ }^{(1)}$.

While discussing the development in electricity no one should overlook the discovery and contribution of these philosophers and scientists: Thales, Stephen Gray (in early $17^{\text {th }}$ century), DuFay (in 1733), Coulomb, Galvani, Volta, Oersted, Ampere, Ohm, Seebeck, Faraday, Henry, Maxwell, Thomson, and Drude.

Electrical conductivity is one of the physical properties of a material which depends on structure of that material and hence on bonds that held the material together ${ }^{(2)}$. In order to choose a material for fabrications and suitability in use we should consider building blocks \& properties of the material, interaction of that material with environment, other materials surrounding it ${ }^{(2)}$ and cost of the material.

\section{INTRODUCTION}

The phenomena associated with the storage of electric charge or motion of the charged particles in specified direction through a conductor is known as electricity. Former electricity due to the charge at rest is called static electricity and latter is called current electricity. Only the electric field is associated with static electricity whereas current electricity produces both electric and magnetic fields. We need to remember that these two fields are interconvertible and like two sides of a coin, due to which our lives are more comfortable and development of the world is accelerating day by day.

Materials present around us are classified mainly into metals, ceramics, polymers, composites and biologics (3) on the basis of their makeup/ arrangement and types of bonds that held the materials together. Electron theory tells that presence of free electrons in a material is accountable for the electrical conduction. Thus, metals are good conductors due to having a large number of free electrons in conduction band whereas non-metals, plastics, rubber etc, with no free electron, are bad conductors/insulators of electricity. Conduction of electricity through a material depends on number of free electrons, impurity and imperfections (4). So, different materials have different capability to conduct the electricity. Ability of a material to oppose the flow of electrons or electric charge is known as resistivity $\rho$. But ability of the material to conduct electricity is conductivity, $\sigma$. These two properties of a material are exactly opposite and reciprocate to each other. In the absence of an external electric field electrons move randomly and no net velocity results for individual electrons and no electric current is developed. If an electric field is applied in a conductor, electrons have finite resultant velocity (drift velocity) in specified direction i.e., 
in the direction opposite to the electric field which results an electric current.

\section{MATHEMATICAL FORMULATIONS}

De Broglie proposed the dual nature of particle like energy, on his thesis entitled Research on the Theory of Quanta in 1924, later awarded his doctorate and won the Noble Prize in $1929^{(5)}$. An electron can have wave nature as well which is known as matter wave.

Matter waves are believed to be scattered by lattice atoms. When the lattice atoms absorb the energy of incident waves then they get oscillated as oscillators and in turn reemit/scatter the radiation in all directions. If these waves from two or more atoms in a periodic crystal are in phase, they superpose and interfere constructively and the resulting wave doesn't suffer any change in intensity and direction i.e. matter waves do not lose energy but may be modified only in velocity ${ }^{(3)}$. This type of coherent scattering is observed in perfect or ideal crystal.

But in real crystal scattering centers are not periodically arranged due to impurity, imperfections and thermal agitations so that the reemitted waves are not in same phase and the wave is said to be incoherently scattered. The energy of incoherently scattered waves gets diminished in forward direction. Thus, the intensity of the resultant wave after reinforce is significantly modified i.e., matter waves lose energy. It is the wave picture of origin of the electrical resistance \& resistivity and at the same time conductance and conductivity.

\section{a. Classical Electron Approach of Conductivity}

When an electric field $\mathbf{E}$ is applied to a conductor having free electron $\mathbf{n}$ in unit volume then the electrons get accelerated and drifted by the force eE towards positive ions/lattice sites. From Newton's second law, we have

$$
m \frac{d v}{d t}=e E
$$

Where, $\mathrm{m}$, e and $\mathrm{v}$ are mass, charge and velocity respectively of the electron.
After removal of the electric field the electron is supposed to move with a constant velocity. Usually it does not happen so ${ }^{(1)}$ due to the presence of resistance caused by the interactions of drifting electrons with lattice atoms, impurity and imperfections. Now as to above consideration equation (1) is modified into

$$
m \frac{d v}{d t}+\gamma v=e E
$$

Where $\gamma$ is a constant called resistive/damping constant and $\gamma \mathrm{v}$ is damping/resistive force.

Due to the interaction of elections and lattice atoms and electric field there is acceleration of electrons between two successive collisions and on the other hand electrons impart energy to the lattice atoms. Finally the electron acquires a constant drift speed $\mathrm{V}_{\mathrm{D}}$ as a result the equation (2) reduces into

$$
\begin{array}{r}
\gamma v_{D}=e E \\
\text { Or, } \gamma=\frac{e E}{v_{D}}
\end{array}
$$

From the equations (2) and (3), we have

$$
m \frac{d v}{d t}+\frac{e E}{v_{D}} v=e E
$$

It is an actual differential equation of motion for an electron in the presence of resistive as well as the electric fields. Solution of this differential equation is as given below:

$$
v=v_{D}\left[1-\exp \left\{-\left(\frac{e E}{m v_{D}}\right) t\right]\right.
$$

This relation shows that velocity, $v$ of the electron depends directly on external electric field and inversely with resistive constant. Moreover the term $\frac{m v_{D}}{e E} \mathrm{~h}$ has the dimension of time called relaxation time, $\tau$ (Hummer, 1985) it is an average time between two consecutive collisions.

$$
\text { i.e., } \tau=\frac{m v_{D}}{e E}
$$

Rearranging above relation, we have

$$
v_{D}=\frac{e E \tau}{m}
$$

From general formula of current density, $\mathrm{J}$ and microscopic view of Ohm's law, we have 


$$
\mathrm{J}=\mathrm{n} \mathrm{e} \mathrm{v}_{\mathrm{D}}=\sigma \mathrm{E}
$$

Where, $\sigma$ is electrical conductivity of the material. After rearranging the above relation and using equation (7), we have

$$
\begin{aligned}
& \sigma=\frac{\ddot{\mathrm{n} e \dot{\mathrm{v}}_{\mathrm{D}}}}{\mathrm{E}}=\frac{\mathrm{n} \mathrm{e}^{2} \tau}{m} \\
& {\left[\because v_{D}=\frac{e E \tau}{m}\right]}
\end{aligned}
$$

It is the classical electron/Drude-Lorentz Equation of electrical dc conductivity. This equation shows that electrical conductivity, $\sigma$ of the material is proportional to the number of free electrons per unit volume (population density) and relaxation time. According to this law, magnesium would be more conductive than copper, contrasts to the fact/ experimental observations, which is one of the drawbacks of this law and it is required to revise.

The mean free path, 1 of a conduction/free electron is defined as ${ }^{(6)}$

$$
1=\mathrm{v}_{\mathrm{D}} \tau
$$

\section{b. Quantum Mechanical Approach of Electrical Conductivity}

As we are known that no net velocity of free electrons in a conductor results in the absence of external electric field but a net velocity of the free electrons in a conductor results in the presence of an external electric field and give rise to an electric current. It can be expressed in velocity space/vector space as shown in fig (1).
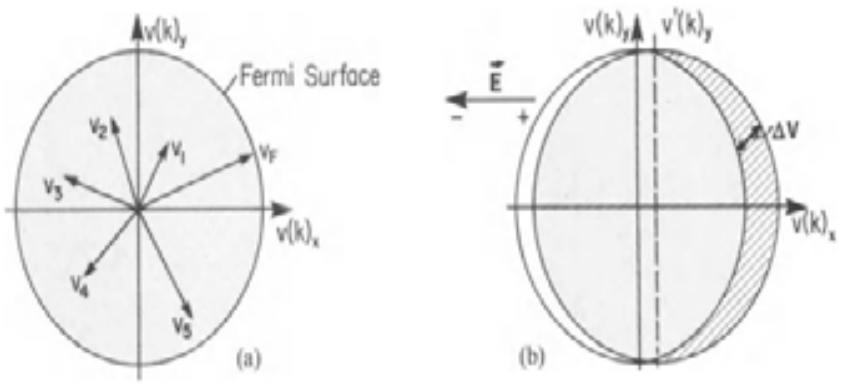

Figure (1). Velocity of electrons in two dimensional space a) without electric field, and b) with the electric field, E. [Courtesy: Hummer, 1985]
In the figure (1) $\mathrm{v}_{\mathrm{F}}$ is Fermi velocity, maximum velocity in velocity space, firmly associated with Fermi energy $\left(\mathrm{E}_{\mathrm{F}}\right)$ which is the maximum kinetic energy of an free electron at absolute zero temperature (7), (8). In the absence of electric field velocity vectors cancelled each other pairwise at equilibrium in Fermi sphere, as shown in figure (1). a, and no net velocity of electron results (1) i.e., no net current results. On the other hand, in the presence of electric field velocity of the majority of the electrons again cancelled each other pairwise but some electrons near Fermi surface gain velocity $\mathrm{v}+\Delta \mathrm{v}$ and Fermi sphere displaces in the direction opposite to the external field in velocity space as shown in figure (1).b. Only these electrons (population density say $\mathrm{n}_{\mathrm{F}}$ ) with velocity $\mathrm{v}+\Delta \mathrm{v}$ actually cause the observed electric current.

Now, the number of electrons they take part in electrical conduction is $n_{F}$ so the current density can be expressed as

$$
\mathrm{J}=\mathrm{n}_{\mathrm{F}} \text { e } \mathrm{v}_{\mathrm{F}}
$$

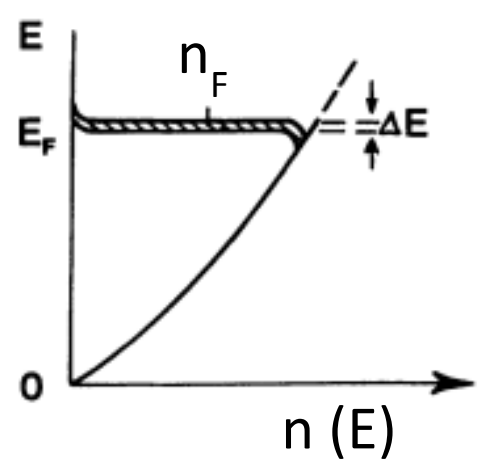

Figure (2). Energy of free electron versus population density. [Courtesy: Hummer, 1985]

The number of electrons having energy slightly greater than Fermi energy due to the external field which yields the electric current is given by relation

$$
n_{F}=n(E) \Delta E=\mathrm{n}(\mathrm{E}) \frac{\mathrm{dE}}{\mathrm{dk}} \square \mathbf{k}
$$

Where, $\mathrm{k}$ is wave vector. Quantum mechanically, we have, energy of a free electron is

$$
E=\frac{\hbar^{2}}{2 m} k^{2}
$$


And next we have

$$
k=\frac{p}{\hbar}
$$

Where, pismomentum of the electron. Differentiating equation (13) and using equation (14), we have

$$
\frac{d E}{d k}=\frac{\hbar^{2}}{m} k=\hbar v_{F}
$$

And again differentiating equation (14), we have

$$
\Delta k=\frac{d E}{\hbar} \Delta t=\frac{d E}{\hbar} \tau
$$

here $\Delta \mathrm{t}$ is time interval between two consecutive collisions $=\tau$, relaxation time.

Now from the equations (11), (12), (15), \& (16), we have

$$
J=v_{F}^{2} e^{2} n(E) E \tau
$$

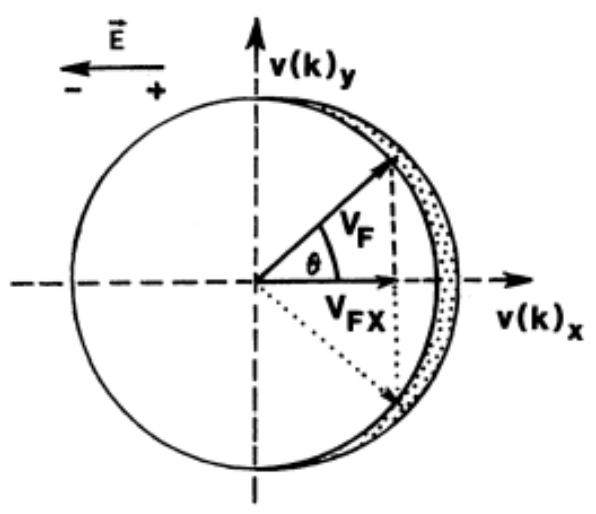

Figure (3). Projection of Fermi velocity along positive $v(k)_{x}$ direction. [Courtesy: Hummer, 1985]

Only the component $\mathrm{v}_{\mathrm{F}} \cos \Theta$ contribute to the electric current as shown in figure (3), so we have

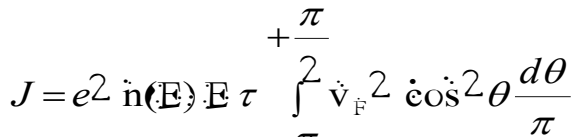

$$
\begin{aligned}
& \text { or, } J=\frac{-\frac{\pi}{2}}{2} e^{2} n(E) E \tau v_{F}^{2} \\
& \text { (for two dimensional space) }
\end{aligned}
$$

Similarly for the spherical Fermi surface, we have

$$
J=\frac{1}{3} e^{2} n(E) E \tau v_{F} 2
$$

And we also have $\mathrm{J}=\sigma \mathrm{E}$

From above relations, we have

$$
\text { or, } \sigma=\frac{e^{2} v_{F}^{2} n(E) \tau}{3}
$$

Thus, in quantum mechanical approach conductivity depends on Fermi velocity, population density of electrons, $\mathrm{n}(\mathrm{E})$ and the relaxation time. Moreover the equation (20) shows that not all free electrons but only the free electrons near the Fermi surface contribute to the electrical conductivity. This equation better explains the greater value of electrical conductivity of copper than that of magnesium even though copper has less valence electron than in magnesium. Also, in metal there are many energy levels near Fermi energy and many electrons available to move to contribute the electrical conduction consequently metals have high electrical conductivity ${ }^{(4)}$.

\section{CONCLUSIONS}

This article, a review, has presented a conception on electrical conductivity, and formulations of conductivity using classical electron \& quantum mechanical approaches. The first approach and formulation in equation (9) assumes that all the free electrons in a material drift in the presence of external electric field and all they contribute to the electrical conduction whereas in quantum mechanical approach, in equation (20), only specific electrons drift with a high speed nearly the Fermi velocity $v_{F}$ have supposed to contribute to the electrical conductivity, $\sigma$. In metal there are many energy levels near Fermi energy and many electrons available to move to contribute the electrical conduction therefore metals have high electrical conductivity. Moreover, the quantum mechanical approach is more rational and accurate to explain and determine the electrical conductivity and hence it is believed to be superior over the classical approach. 


\section{ACKNOWLEDGMENTS}

Author acknowledges the University Grants Commission, Nepal, Prof. Dr. Shekhar Gurung, Prof. Dr. Binod K. Bhattarai, Prof. Dr. Bernabe Merí Soucase, and IOE/TU for their support and constant inspiration.

\section{REFERENCES}

(1) Hummer, R. E., "Electronic Properties of Materials", Springer-Verlag Berlin Heidelberg GmbH, Germany, 1985.

(2) Mitchell, B. S., "An Introduction to Material Engineeirng and Science", A John Wiley \& Sons, Inc, Hoboken, New Jersey, USA, 2004.

(3) Hosford, W. F., "Materials for Enginneers", Cambridge University Press, New York, USA, 2008.
(4) http://en.wikipedia.org/wiki/Electrical resistivity_and_conductivity, cited on 07-022014.

(5) http://en.wikipedia.org/wiki/Louis_de Broglie, cited on 07-02-2014.

(6) Kittle C., "Introduction to Solid State Physics", $5^{\text {th }}$ edition, Wiley Eastern Limited, New Delhi, India, 1993.

(7) Murugeshan, R., "Modern Physics", S. Chand \& Company (Pvt.) Ltd, New Delhi, India, 1986.

(8) http://en.wikipedia.org/wiki/Fermi_energy, cited on 07-02-2014. 